\section{IMPACTO ECONÓMICO DEL OCIO EN EL SIGLO XXI}

\author{
Marta Álvarez Alday \\ Asunción Fernández-Villarán Ara \\ Universidad de Deusto \\ Avda. Universidades, 24 \\ 48007 Bilbao \\ marta.alvarez@deusto.es \\ asun.fvillaran@deusto.es
}

\section{ECONOMIC IMPACT OF LEISURE IN THE 21ST CENTURY}

\begin{abstract}
The twenty-first century has radically changed the concept of leisure. Leisure was conceived to happen only out of working hours. Nowadays we are looking for important experiences during all our lives. The Institute of Leisure Studies of the University of Deusto focuses on the human experience beyond the tangible products. We are witnessing a major cultural change in the developed world where most of the population has turned into a philosophy that could be summed up as "having less, enjoying more". At the same time, the TIC'S revolution has propitiated a deep change in the field of the cultural leisure through new areas as consumer electronics -with its greatest exponent: video games- and virtual communities. Leisure, as a result, has become a significant economic sector with a brilliant future. We are trying to address in this article its impact in terms of production, employment and income.
\end{abstract}

KEY WORDS: Leisure economy; economy of culture; tourism and sports.

\section{EсONOMía Y ocio}

La ciencia económica centrada en la resolución de los dos problemas económicos básicos: la asignación de recursos y la distribución de lo producido, es testigo hoy de un importante cambio. Las sociedades que podríamos considerar desarrollas, fruto de más de dos siglos de un proceso de crecimiento económico sostenido de su producción y renta, se encuentran hoy con una asignación de recursos, que lejos de debatirse entre el clásico "cañones y mantequilla1" destina una parte creciente de recursos a facilitar distintas actividades de ocio. El ocio se ha convertido en un importante motor económico y la ciencia económica se esfuerza en adaptarse a este cambio.

Ya definía Lipsey (1977) la economía como una ciencia que estudia las conductas humanas $y$, como tal, se pue-
RESUMEN: El siglo XXI es testigo de una transformación en el concepto de ocio, con un importante aumento de sus actividades con transcendencia económica. Hasta ahora el ocio se consideraba en los periodos de no trabajo y hoy se buscan experiencias importantes a lo largo de todo la vida. El Instituto de Estudios de Ocio de la Universidad de Deusto centra su interés en la experiencia humana más allá de los productos tangibles. Asistimos a un cambio cultural importante en el mundo desarrollado donde la mayor parte de la población se ha volcado en una filosofía que se podría resumir como "tener menos, disfrutar más". Paralelamente, las TIC's han propiciado una revolución en el campo del ocio: el ocio digital con manifestaciones como los videojuegos o las comunidades virtuales. Todo ello se traduce en un importante impacto económico en términos de generación de producción, empleo y renta que tratamos de abordar en este artículo.

PALABRAS CLAVE: Economía del ocio; economía de la cultura; economía del turismo y economía del deporte.

de entender que abarca todas las actividades que puede realizar la persona, incluyendo lógicamente su actividad cultural, deportiva o viajera en la medida en la que tienen una repercusión económica, en su relación con otros. Sampedro $(2009,240)$ afirma con claridad que "no todo es economía (...) pero sí es cierto que casi todo acto humano tiene un componente económico", que ocurre cuando estos actos se realizan en sociedad. La economía como actividad social presenta aspectos técnicos, jurídicos, psicológicos o culturales, entre otros. Precisamente, un rasgo esencial del Instituto de Estudios de Ocio de la Universidad de Deusto (Cuenca, 2011a) es la aproximación global al ocio, que, al ser entendido como experiencia de la persona y fenómeno de la sociedad, difícilmente puede ser abarcable desde el conocimiento de una sola de las disciplinas académicas tradicionales, pero se nutre de todas ellas para comprender el fenómeno del ocio en su globalidad. 
En el siglo XXI crece la interacción economía y ocio². Pensadores como Heartfield (2000) ya anticipaban que la parte más rica de la humanidad dedicaria una proporción mayor de su gasto al ocio que a la comida, en la medida en que, al crecer los salarios y bajar los precios de los productos básicos, las personas se comportarian como predijo Maslow. Y cada vez es más clara una economía del ocio con nuevas actitudes y nuevas actividades en las que las personas compran experiencias en vez de cosas. En los últimos años hemos sido testigos de un cambio profundo en la estructura de preferencias de los consumidores: con un aumento relativo del gasto dedicado a las experiencias. El valor de la experiencia perdura en la memoria del que la experimenta, no perece en su fugacidad. Y ello hace que sean más valoradas. Las empresas así lo han entendido ${ }^{3}$ y buscan su ventaja competitiva en conseguir una experiencia en el cliente más allá del consumo del bien o del disfrute del servicio (Pine y Gilmore, 2002), pero que requiere necesariamente de la participación personal del adquirente. Desde la perspectiva del ocio se habla del ocio experiencial, que tiene precisamente su fundamento en la implicación personal, desarrollando tanto la capacidad de esfuerzo como la sensibilidad. En palabras de Cuenca (2011b, 86): "es un ocio difícil porque nos enfrenta a retos con nosotros mismos y nos pide un compromiso y acción".

Por otro lado, la innovación tecnológica afecta a la industria del ocio, no sólo a través de la liberación de tiempo y dinero, que se dedicarán a aumentar su demanda, sino que también transforma las actividades de ocio (Landabidea et al., 2011). El cine, la televisión, video canales por satélite y ahora digitales compiten entre sí. Es la era del ocio digital, con un espectro tan amplio como la electrónica de consumo o las comunidades virtuales (Aguilar, 2008). Los videojuegos "se configuran en la actualidad como la actividad lúdica de mayor importancia social en relación al uso de las TIC, tanto por la cantidad de practicantes (la Interactive Software Federation of Europe (2007) estima que el $25 \%$ de la población de Italia, España, Alemania, Francia y Reino Unido juega a videojuegos regularmente) como por el impacto económico de su industria ${ }^{4}$, que según AdeSe (2006) ha sido, en todo el mundo, de 21.000 millones de euros lo que la sitúa como la primera dentro del sector de ocio audiovisual" (Aguilar, 2011, 188). Destaca el ejemplo de España, donde del total del consumo de ocio audiovisual e interactivo, el 35\% del mismo se refiere a los videojuegos, por encima de la taquilla del cine, la música grabada y las películas de video, lo que demuestra la importancia de este sector (AdeSe, 2006, citado en Aguilar, 2011).

Desde una perspectiva económica, el ocio se configura como un sector cada vez más importante en la economía y como una alternativa de desarrollo en regiones con problemas estructurales que ven amenazados sus sectores productivos tradicionales. Sin embargo, en el ámbito económico nos encontramos con una gran dificultad a la hora de cuantificar el impacto económico del ocio. En realidad, ello se debe a que somos herederos de la revolución industrial y nuestras clasificaciones de actividad económica, de estadísticas, etc., están concebidas para una economía en la que el sector industrial era clave. El ocio se definía como tiempo de no trabajo y se utilizaba para coger fuerzas y recuperarse para volver a trabajar. Aunque estos tiempos están lejos para los ciudadanos del mundo desarrollado, que aspiran a tener experiencias importantes de ocio a lo largo de toda su vida ${ }^{5}$, el desarrollo de una metodología común requiere tiempo todavía. Desde un punto de vista conceptual, la mayor parte de las industrias de ocio -a excepción de la fabricación de elementos para la práctica de actividades de ocio- se incluyen en el sector servicios. Este sector representa ya el 70\% del PIB como media en los países de la OCDE y el 62\% en España según los últimos datos disponibles para 1996 (OCDE, 2009).

Las personas de la sociedad moderna tienen más posibilidades para la realización del ocio que ninguna otra persona en ningún tiempo de la historia de la humanidad (Cuenca, 2011a). El crecimiento de las industrias del ocio ha sido más rápido de lo que hubieran necesitado no solo la adecuación de nuestros sistemas de contabilidad nacional sino incluso de la sociedad para madurar y cambiar sus valores. No se puede olvidar que unas poblaciones sin la formación y preparación adecuadas se vuelcan en prácticas de ocio negativas como pueden ser los hábitos nocivos de fin de semana (la industria del alcohol y otras drogas son uno de los grandes negocios mundiales), el consumismo feroz de los adultos (crecimiento de los shopping centers de consumo compulsivo de bienes y comida o el auge de las compras con cupones de descuento en sitios como Groupon, Letsbonus, Notelapierdas, Clubcupón, ClickOn, Elgrupazo y Descuentocity que reciben diariamente decenas de miles de visitas y su acelerado crecimiento ha generado un fuerte incremento en el comercio electrónico) o el aburrimiento de las personas mayores (con un impacto negativo en gastos de sanidad). 


\section{Contribución del ocio a la eConomía}

El impacto económico de las industrias de ocio, en todas sus variedades, es extraordinario porque de alguna forma se han convertido en las protagonistas a futuro de la generación de PIB, renta y empleo en el mundo rico -resultado de la terciarización de sus economías y de los cambios en la estructura de preferencias- y en buena medida también en el mundo pobre -por las exportaciones de bienes y servicios y por entenderse como una solución a corto plazo para salir de la pobreza y a largo como forma de crecimiento económico por las posibilidades de diversificación que puede abrir 6 .

La contribución de las industrias del ocio se podría resumir en:

1. Generación de producción, renta y empleo en los países que producen los bienes, servicios y experiencias (incluyendo su aportación a las arcas del estado vía impositiva).

2. Aumento de exportación (y/o atracción de turistas) con la consiguiente entrada de divisas.

3. Efecto de arrastre, a través de las relaciones intersectoriales, sobre el resto de actividades económicas (con especial relevancia del turismo y de las infraestructuras de ocio).

4. Aumento de las oportunidades de diversificación en el terreno económico.

5. Mejora de la imagen de la región/país ligado a determinadas actividades y por lo tanto de sus posibilidades de atracción de negocios, inversión extranjera y visitantes.

A continuación se analiza la medición y el impacto de las industrias de ocio agrupadas en tres grandes grupos como son el turismo, la cultura y el deporte.

\subsection{Medición}

La Medición de los impactos económicos de las Industrias de Ocio exige una tarea previa de delimitación de dichas industrias. Desde una perspectiva histórica resultan de obligada referencia autores como Dumazadier (1964), que profundiza en los temas de ocio y cultura, Tribe (1999), en ocio y turismo, o Gratton y Taylor (2000), con su estudio del impacto económico del entretenimiento y del deporte.
Dado que el avance es desigual en las distintas áreas se aborda su estudio por separado.

\section{Turismo}

La Comisión de Estadísticas de Naciones Unidas define el turismo como "las actividades que realizan las personas durante sus viajes y estancias en lugares distintos al de su entorno habitual, por un período de tiempo consecutivo inferior a un año con fines de ocio, por negocios y otros motivos" (IET, 2004, 3). El turismo es un sector de actividad transversal en el que conviven servicios y negocios de diversa índole como la restauración, el transporte, los servicios relacionados con el ocio, las empresas deportivas, e incluso el sector comercial y la construcción.

Su cuantificación. Al tratarse no de la producción de bienes y servicios sino del gasto de un determinado tipo de consumidor (turista o excursionista, residente o no) en bienes y servicios, no está clasificado como actividad económica. En España el Instituto de Estudios Turísticos ${ }^{7}$ (IET) es el propulsor, en primera instancia, de la Cuenta Satélite del Turismo (CST) que se presenta como la herramienta más adecuada para la cuantificación de los impactos económicos del turismo. Su objetivo es la medición macroeconómica del turismo siguiendo las recomendaciones de los organismos internacionales, como la Organización Mundial del Turismo (OMT), la OCDE y el Eurostat, dentro del marco de la Contabilidad Nacional de España8.

Tres grandes magnitudes recogen el impacto económico del turismo: el consumo, la oferta de bienes y servicios y el empleo.

El consumo: siguiendo a la OMT (1991) se trata de: "Todo gasto efectuado por el visitante o por su cuenta durante su desplazamiento y estancia turistica en el lugar de destino". Los motivos turísticos que considera la OMT son el ocio, los negocios, visitas de familiares y amigos y tratamientos de salud. Se trata no solo de cuantificarlos sino de conocer su composición en cuanto a la unidad realizadora del gasto: hogares (residentes y no residentes; dentro y fuera del territorio económico nacional); empresas (gastos por cuenta de las empresas); administraciones públicas (viajes de sus funcionarios pagados por la Administración Pública).

ARBOR Vol. 188754 marzo-abril [2012] 351-363 ISSN: 0210-1963 
La oferta de bienes y servicios: la elaboración de la Cuenta Satélite de Turismo exige la previa construcción de las tablas Input-Output (Álvarez, 2003), porque se trata de estimar la parte de producción de determinadas ramas de actividad destinada a cubrir la demanda turística. Ante la dificultad de encontrar una rama de actividad exclusivamente turistica, para su estimación se identifican servicios incluidos en la experiencia turística como transporte, restauración, alojamiento y otros; se estima una renta ficticia a segundas residencias de uso turistico; y se tratan de conocer los bienes inmuebles vinculados, desde la doble perspectiva de actividad de construcción y de bienes de capital producidos.

El mercado laboral interesa no solo en cuanto a número de empleos sino en cuanto a su calidad. En España se estudia desde el lado de la oferta definiéndose como el conjunto de actividades que total o parcialmente producen bienes y servicios que son adquiridos por los turistas, lo que supone dejar fuera el empleo indirecto. Destacan, como principales limitaciones, el hecho de que se incluye el gasto de residentes locales en esos epígrafes y se excluye el gasto de turistas en actividades no características.

\section{Cultura}

La cultura ha sido quizá la dimensión que más se ha resistido a su cuantificación económica (Throsby, 2001). Históricamente el arte o la creación se han revelado ante su posible vinculación con el negocio. Sin embargo, su trascendencia económica ha llevado a los gobiernos de los paises desarrollados a intentar buscar un consenso sobre su valoración y así se han ido desarrollando metodologías específicas, que se adaptan a cada realidad nacional y difícilmente permiten la comparación con otros paises e incluso regiones.

Desde un punto de vista conceptual, Bustamante y Zallo (1998) establecen una primera aproximación a la Industria cultural como conjunto de ramas y actividades auxiliares, productoras y distribuidoras de bienes y servicios con contenidos simbólicos, concebidas por un trabajo creativo, organizadas por un capital que se valoriza y destinadas finalmente a los mercados de consumo con una función de reproducción ideológica y social. A partir de aquí, desde los años 90, diversos estudios de García, Fernández y Zofío (2001), que realizan una revisión de los trabajos de Baumol y Bowen (1966) y Frey (2000), García y Zofío (2006) y en especial García et al. (2009) han avanzado en la construcción de un marco analítico riguroso y sistemático que permite determinar la dimensión y la importancia de lo que denominan industria de la cultura y el ocio en España. La delimitación y valoración económica de esta industria, debe abarcar así tanto su oferta privada como la provista por el sector público. García, Fernández y Zofío (2001:19) distinguen dos subsectores ${ }^{9}$ y posteriormente García et al. (2009) realizan una comparativa de lo que se considera la industria de la cultura y del ocio en Europa, Estados Unidos, U.K. y España partiendo de la información disponible que proviene tanto de consultoras privadas como de instituciones gubernamentales, y que permite afirmar que no hay una consideración única del concepto y por lo tanto no se pueden hacer comparaciones entre países. En esencia, el resumen de sus hallazgos se resume a continuación:

I. Sólo se consideran como industrias de la cultura y el ocio en todos los paises:

1. En el ámbito de la cultura:

- Artes EMA (Escénicas, musicales, cine y video, televisión y radio)

- Artes plásticas (Pintura y Escultura, Fotografía)

- Edición e impresión de libros (Libros, Prensa Escrita)

2. En el ámbito de la Creatividad: la Publicidad.

II. Diferencias entre países:

1. En cultura: Museos y Bibliotecas son considerados por todos excepto por el Reino Unido; Patrimonio histórico es considerado por todos excepto por EE.UU. La política lingüística y la juventud sólo se consideran actividades de la industria cultural en España.

2. En creatividad: la arquitectura, el diseño y el software son considerados como industrias por EE.UU., la Unión Europea y el Reino Unido; no así por España. La gestión de derechos no se considera ni en España ni en el Reino Unido.

3. En el epígrafe de Ocio, España considera actividades de la Industria del ocio y de la cultura tanto las deportivas, taurinas, parques recreativos, ferias y 
salones, loterías y apuestas y juguetes. Sin embargo, EE.UU. sólo considera los juguetes; Reino Unido sólo las actividades deportivas y la Unión Europea los parques recreativos y las ferias y salones.

4. Por último, en lo que son Industrias de apoyo, EE.UU. considera el comercio mayorista y minorista, el transporte y las telecomunicaciones. Europa y Reino Unido no consideran ninguna de las tres y España solo el comercio minorista y mayorista.

Finalmente, conviene constatar la dificultad para definir los ámbitos que componen la cultura como sector y su heterogeneidad productiva al reunir en su seno actividades industriales junto a actividades artesanales y a un largo número de servicios. La dimensión y trascendencia económica y social de cada uno de ellos es muy dispar. Así, pues, no es extraño que se disponga de más información sobre los subsectores industriales más tradicionales (libro, cine), o con una mayor presencia de la administración pública (bibliotecas, archivos, museos), que sobre los más nuevos (vídeo, multimedia) o artesanales (artes plásticas, artes escénicas). Existen múltiples aproximaciones metodológicas y analíticas posibles, desde la antropológica a la estética, pasando por la sociológica, la politológica o la económica. Todas ellas enriquecen el conocimiento del sector, pero su peso no es homogéneo en el momento del enfoque e hipótesis del trabajo estadístico convencional (Bonet, 2004). Finalmente, disponer de datos comparables a nivel internacional ha sido, tradicionalmente, un reto perseguido por investigadores y gobernantes, pues permite comparar realidades y situaciones diversas. Sin embargo, el Instituto de Estadísticas de UNESCO, que desde los años setenta nutría de referentes comunes, ha decidido abandonar la recogida sistemática y la publicación de sus series históricas ante la imposibilidad de crear un sistema universal de indicadores de la realidad cultural planetaria. Así se lo han dicho buena parte de los especialistas en análisis comparado, muy a pesar de las implicaciones prácticas que comporta (UNESCO, 2002), pues, sigue siendo necesario disponer de datos para intentar comparar e interpretar la realidad, y la evolución de los distintos sectores y manifestaciones culturales.

En España (MCU, 2008), el Ministerio de Cultura optó en 1995 por elaborar una publicación ${ }^{10}$ "Cultura en Cifras" en una tarea de recopilación y organización de los datos existentes sobre la cultura que significó un hito importante para el conocimiento de la situación del sector. La siguiente publicación fue "Las cifras de la cultura en España. Edición 2002" que recogía la evolución de las cifras en la década anterior, y presentaba la situación de la cultura en las diferentes Comunidades Autónomas, y trataba de disponer, en la medida de lo posible, de datos homogéneos que permitieran una comparación internacional. El siguiente trabajo es el "Anuario de estadísticas culturales 2005", que se ha establecido con periodicidad anual. El Anuario del 2009 incorpora por primera vez un bloque con las estadísticas de síntesis donde se incluye un avance de la Cuenta Satélite de la Cultura con la finalidad de estimar el impacto global de la cultura en el conjunto de la economía española. Así, "El anuario de estadísticas culturales 2010", su sexta edición, es el último disponible (MCU, 2010).

\section{Deporte}

Según evidencia la Comisión de las Comunidades Europeas $(2001,2)$ : "Un ciudadano europeo de cada tres practica regularmente una actividad física". El deporte se ha convertido en uno de los fenómenos sociales más sobresalientes de Europa durante el siglo XX y así lo reflejan los datos sobre el número de practicantes, el interés de los ciudadanos, su inclusión en las políticas públicas y sus repercusiones económicas. Los Estados miembros de la Unión Europea figuran entre las principales potencias deportivas mundiales y el territorio comunitario acoge un número cada vez más elevado de acontecimientos deportivos.

También en España, como actividad de tiempo libre, el deporte se ha convertido en un pasatiempo muy apreciado y ha pasado a ser un importante producto de consumo de masas, tanto como manifestación de espectáculo deportivo omnipresente en los medios de comunicación, como consumo de artículos deportivos para su uso personal y familiar (García, 2006).

El deporte se está configurando como un elemento clave de las sociedades modernas, entre otras razones, por su contribución al desarrollo económico y social y por su sinergia con la educación, la cultura, la salud y el turismo.

La consideración del deporte como un bien desde la perspectiva económica es algo novedoso que se ha intensificado en los últimos años. Sin embargo, a la hora de medir la importancia de este sector en la economía no 
encontramos estadísticas fiables y sistemáticas, ni siquiera existe un consenso sobre las acepciones y los tipos de actividades que configuran el ocio deportivo (Pedrosa y Salvador, 2003).

La investigación sobre la aplicación de conceptos económicos en actividades deportivas se ha multiplicado en las últimas décadas: ya sea la contribución de deportes al crecimiento económico, la competencia sobre los derechos de los medios de comunicación o los mercados de trabajo de estrellas del deporte. Los trabajos de investigación reflejan las concepciones contrapuestas del término, tal y como afirma Lera (2010). Así, en Estados Unidos el deporte queda reducido a la práctica deportiva organizada (competición, animación, etc.). Por el contrario, en el ámbito europeo, su concepción es mucho más amplia, englobando a actividades físicas y deportivas que puedan ser realizadas de manera individual y no organizada.

Como describen Andreff y Szymanski (2006) el interés por la economía de los deportes se remonta a $1956^{11}$. Los primeros trabajos abordaban la economía del deporte desde una perspectiva microeconómica y la mayoría se han centrado en la relación entre el deporte profesional y el mercado laboral. Paulatinamente, los estudios se van ampliando a otros campos como el análisis de la demanda, patrocinios, análisis antitrust o subvenciones, siempre desde un punto de vista microeconómico (Heinneman, 1998 y Gratton y Taylor, 2000 y Gratton, 2004).

En la actualidad, la mayoría de los autores consideran tres tipos de actividades deportivas que nos permitirán un análisis de su impacto en la economía: las actividades deportivas personales, las actividades deportivas profesionales y los grandes eventos deportivos.

Su cuantificación. Al igual que en el caso del turismo, tres grandes magnitudes recogen el impacto económico del deporte: el consumo, la oferta de bienes y servicios y el empleo.

El consumo: teniendo en cuenta las diferentes actividades que incluye el deporte a efectos económicos, tendríamos que diferenciar entre dos tipos de gasto. En primer lugar, se encuentra el denominado gasto en deporte activo que se refiere a las actividades deportivas personales y profesionales e incluye gastos de naturaleza tan diversa como la cuota o abono a una sociedad deportiva, club o gimnasio, los gastos de desplazamiento, la compra de calzado y ropa deportiva, la compra de bebida y comida o los servicios de masaje y rehabilitación. Por otro lado, hay que considerar el gasto deportivo pasivo que hace referencia a los espectáculos deportivos e incluye gastos en eventos y abonos, gastos de desplazamiento, alimentos y bebidas, gastos en quinielas y apuestas deportivas, libros, periódicos y revistas especializadas o pagos por eventos en televisión (Junta de Andalucía, 2008).

La oferta de bienes y servicios: Las Cuentas Satélite aportan indicadores sobre la importancia del deporte en el conjunto de la economía.

El primer intento riguroso de medir la importancia económica del deporte es el realizado por Malenfant-Dauriac en 1977 (Junta de Andalucía, 2008). La mayoría de los países europeos han tratado de elaborar una contabilidad nacional de la economía del deporte a imagen de la existente en torno al turismo. Son ya clásicos los informes "Jones", en 1989, y el posterior de "Andreff" (citados en Junta de Andalucía, 2008), realizados por iniciativa del Comité de Desarrollo del Deporte del Consejo de Europa, que han puesto de manifiesto las numerosas dificultades metodológicas que es preciso superar para estimar las principales magnitudes macroeconómicas del deporte. Los primeros intentos europeos de elaborar una contabilidad nacional del deporte se han basado implícita o explícitamente en el modelo que Andreff (en Pedrosa y Salvador, 2003) denomina "tabla económica de conjunto", que describe tres tipos de operaciones económicas (de producción, de reparto de rentas y financieras), lo que permite describir el equilibrio macroeconómico.

Se han hecho muchos estudios especificos sobre análisis de impactos económicos de determinados eventos deportivos. La metodología difiere según se trate de grandes eventos, como unos Juegos Olímpicos o de campeonatos internacionales, nacionales o locales. El cómputo de los diversos impactos a considerar no está exento de controversia. Ante todo, las magnitudes a estimar dependen, entre otras cosas, del tipo de evento deportivo y de la finalidad del análisis. En el caso de grandes eventos deportivos, como los Juegos Olímpicos, se pueden tener en cuenta multitud de factores, entre los que cuentan, por ejemplo, las inversiones específicas en infraestructuras 
de transportes, en instalaciones deportivas, en infraestructuras turisticas, etc.; asimismo el impacto sobre la actividad del sector turístico cobra en estos casos una especial relevancia, por lo que suele ser también objeto de evaluación.

Para medir el impacto del deporte en la economía, directores de deporte de la UE, en su reunión en Viena en marzo de 2006, decidieron establecer el grupo de trabajo "Deporte \& economía" para desarrollar un enfoque común, incluyendo un método estadístico para medir el impacto económico del deporte en la UE. En particular, el grupo de trabajo ha encontrado consenso sobre una definición común, la "Definición Vilnius del deporte", como base para la recopilación y la producción de datos a nivel nacional y, por tanto, la recopilación de cuentas nacionales satélite de deporte (Sport Satellite Account-SSA). Una cuenta satélite de deporte (SSA) permite que todas las actividades económicas relacionadas con el deporte aparezcan explícitamente, en lugar de mantenerlos ocultos en las cuentas nacionales. En estos momentos, únicamente se dispone de resultados para tres de los países implicados en el desarrollo de estas cuentas satélites del deporte: Reino Unido, Austria y Chipre (SpEA, 2010).

El empleo: Dentro del impacto económico provocado por el deporte se analizan también los efectos producidos en términos de generación de empleo. El principal problema metodológico gira en torno a la definición de las actividades incluidas dentro de este subsector. En este marco nos encontramos con estudios que consideran los empleos generados por los servicios directamente relacionados con la práctica deportiva; en otros casos, el análisis se limita a la enseñanza, entrenamiento, animación y dirección de instalaciones deportivas. En otras ocasiones, se incluyen todas las actividades económicas relacionadas con el deporte incluyendo algunas tan diversas como la industria de la ropa deportiva, la alimentación o los medios de transporte y hostelería relacionados con el deporte.

En cualquier caso, sea cual sea la definición de deporte utilizada, todos los autores coinciden en afirmar el importante incremento producido en los últimos años, motivado, entre otras razones, por el acceso de nuevos colectivos a la práctica del deporte (minusválidos, tercera edad, etc.) y por los cambios en los estilos de vida de las sociedades desarrolladas.
El voluntarismo sigue siendo otra de las características que marcan a este sector sobre todo en el ámbito asociativo de clubes y federaciones, aunque se observa una tendencia creciente en la profesionalización del empleo alrededor del deporte, sustituyendo progresivamente al voluntariado que históricamente ha atendido este sector.

\subsection{Impactos}

\section{Turismo}

Se trata de la primera actividad en ser cuantificada desde el punto de vista económico.

Impulsado por la mejora de las condiciones económicas en todo el mundo, el turismo internacional se ha recuperado más rápido de lo esperado de los efectos de la crisis financiera mundial y la recesión económica de finales de 2008 y 2009. Las Ilegadas de turistas internacionales se incrementaron un 6,7\% en comparación con 2009, con un crecimiento positivo en todas las regiones del mundo. El número de llegadas alcanzó 935 millones, superando en 58 millones la cifra de 2009 y en 22 millones el nivel máximo de 2008 previo a la crisis (913 millones).

Esta misma tendencia se observa en España, que fue, en 2010, el destino elegido por 52,7 millones de turistas internacionales. De esta forma, el año 2010 se convierte en un año de transición en el que cambia la tendencia al romper dos años consecutivos de descensos y abre una etapa que se presume de sostenida recuperación (IET, 2009, 2010, 2011).

En la aproximación a la evaluación de la importancia del turismo en España, cabe destacar que, a diferencia de la década anterior, donde las estimaciones de la anterior serie de la CST evidenciaban un incremento del peso del turismo en la economía (Álvarez, 2006), desde el año 2000 se observa una paulatina y constante pérdida de peso del turismo en el total de la economía, que se ha traducido en la disminución de la aportación de las actividades turísticas al producto interior bruto, que pasa de más de un $11,5 \%$ a principios de la década hasta el 10\% en el último año del que se disponen de mediciones (2009).

A pesar de estos datos, el turismo sigue constituyendo una de las actividades económicas de mayor calado en el pa-

ARBOR Vol. 188754 marzo-abril [2012] 351-363 ISSN: 0210-1963

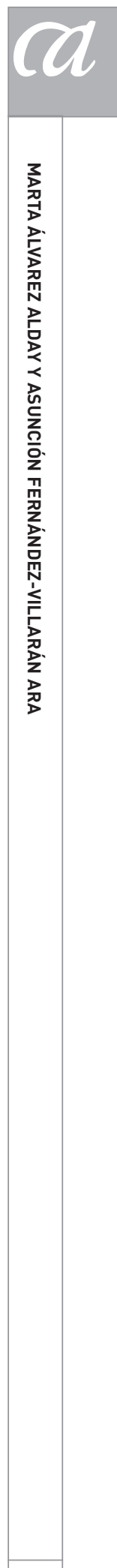

357 
norama nacional, llegando a constituir la principal fuente de generación de renta en algunos municipios españoles. En este sentido cabe destacar que el turismo proporciona empleo a cerca de 2.500 .000 personas, lo que representa el $15,8 \%$ del empleo en los servicios ${ }^{12}$.

En los dos últimos años, España, que ha desarrollado otros atractivos al margen del sol y playa (Fernández-Villarán y Rodríguez, 2004), ha perdido su posición en el panorama mundial y ocupa el cuarto lugar entre las preferencias de los turistas, por detrás de Francia, Estados Unidos y China. En el ejercicio 2010 llegaron a España algo más de 52,7 millones de turistas extranjeros (el 5,6\% del mercado mundial).

La aportación de divisas turisticas continúa resultando una ayuda importante con la que compensar el tradicional déficit comercial español: el saldo de la sub-balanza de turismo y viajes del ejercicio 2010 permitió una tasa de cobertura del $58 \%$, con una sustancial mejora respecto al año 2008 donde dicha tasa de cobertura era del 32\% y contribuyendo, en definitiva, al desarrollo económico y a la modernización del país.

\section{Cultura}

La dimensión económica de la industria de la cultura es cada vez mayor en unas sociedades desarrolladas en las que la demanda de productos culturales y de entretenimiento crece al instalarse en los hábitos de consumo de los ciudadanos, con un crecimiento exponencial y absolutamente nuevo del ocio digital nacido de la interacción con las nuevas TIC, porque como apunta Kozinets (2010) muchos mundos sociales se están tornando digitales. En el año 2008 (INE, 2008), el $99,7 \%$ de los hogares españoles disponía de televisión y el DVD estaba presente en tres de cada cuatro hogares, en detrimento del vídeo. El $63,6 \%$ de los hogares con al menos un miembro de 16 a 74 años disponía de algún tipo de ordenador, y el 51,0\% tenía acceso a Internet ${ }^{13}$. Las nuevas tecnologías han propiciado la aparición de nuevas fórmulas para el entretenimiento. La mensajería instantánea (messenger) es utilizada por el $53,8 \%$ de los internautas, un $52,4 \%$ descarga y/o escucha música por la red y un $41,6 \%$ descarga y/o ve películas o vídeos por Internet.

La diferente delimitación del sector de la cultura hace muy difícil llegar a una única valoración. La ausencia de una metodología común dificulta, además, la obtención de información agregada por países. Cabe señalar que en los últimos años en España (INE, 2008) el gasto de los hogares en ocio se ha incrementado en algunos bienes $y$ servicios (vacaciones todo incluido, servicios recreativos y deportivos), mientras que ha disminuido en otros (prensa, juegos de azar). En paridades de poder adquisitivo entre los paises, el gasto total de los hogares españoles en recreación y cultura se sitúa por debajo de la media de la Unión Europea y muy por encima en lo que se refiere a gastos en restaurantes y hoteles. El cine es la actividad cultural preferida, junto con la visita a lugares de interés. Ver la tele y cultivar la vida social son otras actividades de ocio a las que se dedica gran parte del tiempo libre.

En relación al empleo $(I N E, 2008)$ se trata de un empleo estable pero con alta formación. En el año 2005 el empleo cultural representa un $2,4 \%$ del total de empleo en la Unión Europea con una tasa de temporalidad en torno al 16,4\%. En España supone el 2,1\% del total de empleados, con una tasa de temporalidad que alcanza el 29,8\%. Otra caracteristica relevante del empleo en el sector cultural es la alta proporción de trabajadores con educación superior, un $47,7 \%$ en el conjunto de la Unión Europea y un 59,9\% en el caso de España.

Atendiendo a la consideración de actividades culturales del Ministerio de Cultura (MCU, 2010) el empleo cultural sobre el total de empleo en España era el 2,7\% en 2005 y va creciendo hasta situarse en $2,9 \%$ para 2009. $Y$ en relación a la contribución al PIB, en su Cuenta Satélite de la Cultura en España recoge una aportación de las actividades culturales en torno a un 3\% de media anual para el período que va de 2000 al 2009 con una ligera caída $(2,9 \%)$ los dos últimos años.

García et al. (2009), completando una investigación anterior (García, Fernández y Zofío, 2001) que ya situaba al sector cultural y del ocio entre uno de los más dinámicos de la economía española, elaboran sus propias cifras. De sus investigaciones se constata que la industria de la cultura y del ocio, como ellos la delimitan y se recoge en este artículo, viene aportando el 3,6\% del PIB en términos reales en 1997, con valores semejantes los años siguientes llegando al 3,8\% en términos reales en 2003 (último año registrado).

En cualquier caso la aportación al PIB según la distinta delimitación de actividades a considerar oscila entre el 3,8\% 
del PIB para el 2003 (con una consideración ampliada de industrias de cultura y ocio de García et al.) y un 3\% anual de media para el período 2000/2009 del MCU (2010). En relación al empleo si bien el INE establece para 2005 un $2,1 \%$, el MCU lo fija en un 2,7\%, mientras que Garcia et al. llegan a un 7,8\% en 2003. Estos datos nos llevan a afirmar que la industria de la cultura y el ocio se encuentra entre las seis primeras actividades productivas en España.

\section{Deporte}

Los datos disponibles sugieren que el impacto del deporte en la economía está creciendo debido a la diversificación de actividades deportivas, la sociedad de ocio en desarrollo y también la profesionalización y comercialización del deporte. El deporte constituye un importante sector económico en plena expansión, contribuyendo de manera considerable al crecimiento y al empleo. El sector del deporte genera en torno a un 2-3\% del PIB mundial (Lera, 2010).

A nivel del conjunto de la Unión Europea, Dimitrov et al. (2006) estiman en un 3,7\% del PIB europeo la importancia del deporte, incluyendo tanto su impacto directo como indirecto y las competiciones y los grandes acontecimientos deportivos ofrecen amplias posibilidades de desarrollo del turismo en Europa (Com, 2007, 2011).

Para los paises que han desarrollado sus cuentas satélite del turismo, las estimaciones alcanzan en el Reino Unido el $2,6 \%$ del PIB, el $2,4 \%$ en Chipre y el $4,0 \%$ en Austria (Lera, 2010).

De acuerdo con la Encuesta de Hábitos Deportivos de los Españoles (García, 2010), en 2010, cerca de 15 millones de personas de las mayores de 14 años practican algún tipo de deporte, preferiblemente en lugares abiertos y por su cuenta. España cuenta con 3.500.000 licencias federativas. El empleo directamente relacionado con el deporte representa el 2,1\% del empleo total (incluye el voluntariado que casi supera al de empleados).

\section{Conclusiones}

En primer término hay que reconocer la imposibilidad de dar unas cifras agregadas del impacto del ocio en la eco- nomía a nivel mundial o de conjunto de paises e incluso a nivel nacional según los paises, dado que no existe una metodología común aceptada y utilizada por todos. Partiendo de las reflexiones recogidas en Mendoza (2011) sobre la situación de la estadística para el ocio cultural, la hacemos extensible a los otros sectores y podemos afirmar que nos encontramos ante una realidad heterogénea. La situación europea no es extraordinaria, aunque en los últimos años se ha hecho un considerable esfuerzo de sistematización, transparencia y puesta en común. Más allá de la particular situación regional, la información estadística disponible sobre el sector de ocio es escasa (salvo en el caso del turismo), con limitadas series temporales, poco homogéneo país a país, y con una muy baja capacidad para ajustarse a las nuevas necesidades informativas del mundo contemporáneo. Los gobiernos y sus instituciones con responsabilidad o fondos para llevarlas a cabo (institutos de estadistica, bancos centrales, ministerios) tienden a concentrarse en las grandes magnitudes económicas y sociales o bien en aquellos indicadores requeridos desde las instituciones intergubernamentales. Aspectos del ocio, como la cultura, en general, no forman parte de ellos. Destacamos, no obstante, el creciente esfuerzo registrado en este sentido (con ejemplos como el del Ministerio de Cultura español).

Sin embargo, el impacto del ocio en la economía es una evidencia que abarca un campo amplio, en el que se incluyen entretenimiento, deporte, recreación, turismo, cultura, la ropa para la práctica de actividades de ocio, la medicina deportiva, el calzado deportivo, los hoteles, los viajes, los centros deportivos, los centros comerciales, el exponencialmente creciente sector de la electrónica de consumo y el ocio digital y un largo etcétera, que como hemos visto no es objeto de medición homogénea por ningún país.

Si consideramos el problema desde el punto de vista del gasto, claramente el sector del ocio se ha convertido en una actividad económica fundamental. El departamento de comercio de Estados Unidos incluye en este campo los gastos en electrónica para el hogar, radio y televisión, música, juguetes, jardinería, libros y revistas, y equipamiento para el ocio (barcos, bicicletas, etc.). En 2008 el gasto en consumo final de los hogares en $\%$ de su PIB en ocio y cultura supuso el 6,5\% en EE.UU., el 5,2\% en España y una media de un 5\% para los paises de la OCDE (González y Benavides, 2010). Aun así, la mayor parte del gasto en ocio

ARBOR Vol. 188754 marzo-abril [2012] 351-363 ISSN: 0210-1963

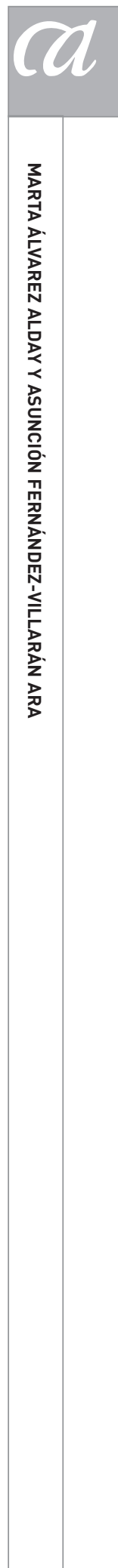

359 
corresponde a otros epígrafes como el transporte, los viajes, el alojamiento, la ropa, la comida e incluso la educación.

En relación a su contribución al PIB en España en 2009, y a pesar de las diferencias señaladas, se estima que el turismo representa un 10\% del PIB, la cultura un $2,9 \%$ y el deporte un 2,6\%.

Es, sin duda, en relación al empleo, donde el ocio despliega su gran potencial. Se podría considerar que hay algún empleo ligado al ocio en prácticamente todos los sectores de la economía (desde la fabricación de zapatillas de deporte o microchips hasta los escritores, artistas, pasando por los atletas profesionales o el pescador que acaba vendiendo su producto a un restaurante). Aun cuando hay una apuesta fuerte en el mundo desarrollado por las Industrias del ocio consumista centrado en los shopping malls ${ }^{14} 0$ grandes superficies comerciales, que desde Estados Unidos se han exportado al mundo entero, o a través de los sitios web de compra con cupones descuento ${ }^{15}$, porque generan empleo no solo en los paises en los que se realiza la comercialización sino en todos aquellos paises de mano de obra barata donde se producen los bienes intercambiados, se constata también un importante desarrollo sociocultural reflejado en el aumento de las actividades de ocio aquí analizadas. El empleo en España debido al turismo, a las industrias culturales y de ocio y al deporte representa una parte creciente del empleo total.

A pesar de no disponer de suficiente información e incluso del hecho de que la información disponible no sea comparable, el trabajo desarrollado en este artículo nos permite afirmar que el ocio se ha convertido en un sector clave de generación de producción y renta y una extraordinaria oportunidad de generación de empleo con un gran potencial a futuro en el mundo entero.

\section{NOTAS}

1 En reflejo de la exigencia a la hora de elegir entre la producción de bienes de consumo o de inversión ante la extraordinaria limitación de recursos que no permitía cubrir todas las necesidades.

2 Graham T. Molitor, presidente de la Public Policy Forecasting y vicepresidente del Word Future Society de Estados Unidos, predecía en 1999 que los negocios orientados al ocio, desde los bares a los teatros de ópera supondrian el 50\% del PNB de los Estados Unidos hacia el 2015 (Molitor, 1999, 14) y que los conglomerados industriales de "big entertainment" como el cine, la televisión, editoriales, música, hoteles o parques temáticos, serían la clave de este sector, como la realidad está demostrando.

3 Empresas como Apple han sido capaces de revolucionar la industria musical mediante su concepto de personalización e integración de la experiencia, pasando de ser una empresa de productos informáticos a una empresa de lo que ellos mismos denominan electrónica de consumo (Aguilar, 2011).

4 Además, los videojuegos, en su permanente proceso de innovación en soportes y juegos han impactado en otros sectores. De hecho, en la actualidad todos los operadores de telefonía móvil están desarrollando servicios de valor añadido que tienen que ver con servicios de ocio, entre los que destacan los videojuegos, descarga de series de TV, acceso a TV y servicios relacionados con la industria musical (SGAE, 2006, citado en Aguilar, 2011).

5 Incluso es posible disfrutar de ocio dentro del tiempo dedicado a la realización de obligaciones tal y como algunas empresas como Google están tratando de promover (Aguilar, 2011) confirmando la postura de Csikszentmihalyi y LeFevre (1989) para quienes
Aceptado: 21 de julio de 2011 
el disfrute puede darse en cualquier actividad o contexto de la vida incluido el trabajo.

6 Los países pobres han visto por ejemplo al turismo como una solución a corto plazo para salir de la pobreza y, a largo, como una promesa de crecimiento económico por las posibilidades de diversificación. La contrastación real después de diversas experiencias sugiere, sin embargo, que, sin una planificación estratégica coherente, el resultado probable son unas infraestructuras deterioradas, una inestabilidad política y social, un control y dependencia externas y un bajo efecto de arrastre sobre sus propias economías (Díaz y Galindo, 2001; Álvarez, 1999). Si el país receptor permite una sobreexplotación de su ecosistema, el deterioro de su medioambiente llevará a una pérdida de calidad del entorno y, por lo tanto, de su producto turístico.

7 Las fuentes más importantes de información son la OMT, la OCDE y el World Travel \& Tourism Council a nivel mundial. A nivel europeo el EUROSTAT y en España el INE, el Banco de España y la Administración General de Turismo que para su coordinación ha creado el IET.

8 Los organismos que intervienen en su elaboración son el Instituto Nacional de Estadística, el Banco de España y el Instituto de Estudios Turísticos. Se encuentra disponible la serie contable 2000-2009.

9 a) Actividades culturales de mercado realizadas por el sector privado, que a su vez se dividen en tres tipos: tipo I o directas que son aquellas sujetas a copyright y por lo tanto susceptibles de generar derechos de copyright, tipo II o indirectas que se asocian a la distribución de las creaciones culturales y de ocio y las tipo III también indirectas pero que representan vehículos de transmisión indispensables para los bienes y servicios culturales y de ocio.

b) Las actividades culturales de ámbito público exigen considerar los distintos sectores institucionales que realizan estas actividades así como el tipo de actividad que realizan. Por institución se agrupan en tres bloques según sean llevadas a cabo por: Estado, Comunidades Autónomas y Corporaciones Locales. Y por naturaleza de actividad, siguiendo los criterios de La Contabilidad Nacional, se pueden clasificar en dos: actividades culturales que se corresponden con la provisión sin ánimo de lucro de bienes y servicios de un marcado carácter social (como una biblioteca) o/y el sector público empresarial cuyas actividades se corresponden con un perfil mercantil, por lo que su valoración se realiza conjuntamente con la del sector privado al estar sujetas a tributación. En resumen su impacto económico se desdoblaría en dos: el de ámbito privado en el que se incluye la actuación empresarial del sector público y las actividades culturales realizadas por el sector público-administraciones públicas-función 45 dentro de la producción de bienes públicos de carácter social -recogido en los presupuestos de las diversas administraciones: estados, comunidades autónomas y corporaciones locales.

10 En la delimitación del ámbito sectorial considerado como punto de partida se tuvieron en cuenta los trabajos metodológicos desarrollados en la Unión Europea (UE) desde el año 1997, en los cuales España participó activamente, que tuvieron como uno de sus principales objetivos la definición de un ámbito cultural común, en la medida de lo posible, a todos los países europeos. Sin embargo, hay diferencias fundamentalmente sobre determinados sectores en los que no existía entre los países un consenso generalizado para su inclusión.

11 Fecha en la que Simon Rottenberg de la Universidad de Chicago escribe un artículo sobre el mercado laboral de los jugadores de baseball.

12 Como rasgos básicos del empleo en España, cabe señalar su estacionalidad, un empleo vinculado al nivel de actividad (contratos temporales y a tiempo parcial), una rotación de personal elevada (baja productividad y calidad del servicio prestado) y el empleo de jóvenes y mujeres por su precariedad.

13 Aun por debajo del promedio registrado en los paises miembros de la UE, que según datos de Eurostat es del 60\%.

14 En este sentido se podría dar como dato la inauguración en abril de 2011 de Marineda City en La Coruña, el centro comercial y de ocio más grande de España y tercero de Europa con una superficie construida de 500.000 $\mathrm{m}^{2}$ y con una generación de empleo directo de 4.500 personas.

15 Como revela el informe Nielsen (2011), publicado con motivo del Día Mundial de Internet, el comercio electrónico es la segunda categoría que más ha crecido en la Red en los últimos meses después de las redes sociales y señala el espectacular aumento de los portales de cupones de descuentos, cuyas audiencias se han disparado en un $68 \%$ en el último año con casi ocho millones de usuarios.

\section{BIBLIOGRAFÍA}

ADESE (2006): Anuario 2005. Accesible en www.adese.es/pdf/anuario-me- 
moria-2005.pdf. Última consulta 14/05/2011.

Aguilar, E. (2008): "Los videojuegos y las comunidades virtuales. Dos ejemplos de ocio digital", Documentos de Estudios de Ocio, núm. 35, Bilbao: Universidad de Deusto, págs. 267-285.

Aguilar, E. (2011): "Ocio e innovación en las organizaciones", Documentos de Estudios de Ocio, núm. 42, Bilbao: Universidad de Deusto, págs. 141196.

Álvarez, M. (1999): "Impacto económico del turismo en el primer y tercer mundos: La alternativa del Ecoturismo", 6. ${ }^{\circ}$ Congreso Mundial de Ocio, Universidad de Deusto, Bilbao.

Álvarez, M. (2003): Introducción a la Estructura Económica, Bilbao, Descleé de Brouwer.

Álvarez, M. (2006): "Economía del ocio", Documentos de Estudios de Ocio, núm. 31, Bilbao: Universidad de Deusto, págs. 21-40.

Andreff, W. y Szymanski, S. (2006): Handbook on the Economics of Sport, Paris: Department of Economics, University Paris 1 Panthéon Sorbonne, France.

Baumol, W. J. y Bowen, W. G. (1996): Performing Arts: The Economic Dilemma, Cambridge, MIT.

Bonet (2004): "La excepción cultural", Real Instituto Elcano de Estudios Internacionales y Estratégicos, págs. 1-8.

Bustamante, E. y Zallo, R. (1998): Las industrias culturales en España, Madrid, Akal.

Csikszentmihalyi y LeFevre (1989): "Optimal experience in work and leisure", Journal of Personality and Social Psychology, vol. 56, núm. 5, págs. 815822.

Comisión de las Comunidades Europeas (2001): Propuesta de Decisión del Parlamento Europeo y del Consejo por la que se establece el Año europeo de la educación por el deporte 2004, pre- sentada por la Comisión, COM (2001) 584 final, Bruselas, 16.10.

Comisión de las Comunidades Europeas (2007): Libro blanco sobre el deporte, Bruselas, COM (2007) 391, de 11.7.2007.

Cuenca, M. (2011a): "La fuerza transformadora del Ocio", Documentos de Estudios de Ocio, núm. 42, Universidad de Deusto, Bilbao.

Cuenca, M. (2011b): "Innovar y aprender disfrutando", Documentos de Estudios de Ocio, núm. 42, Universidad de Deusto, Bilbao.

Díaz, M. A. y Galindo, M. A. (2001): "Una brecha amplia: cultura y desarrollo económico", en Información Comercial Española revista de Economía, núm. 792, págs. 31 a 41.

Dimitrov, D.; Helmenstein, C.; Kleissner, A.; Moser, B. y Schindler, J. (2006): Die makroökonomischen effekte des sports in Europa, Studie in Auftrag des Bundeskanzleramts, Viena: Sektion Sport.

Dumazedier, J. (1964): Hacia una civilización del ocio, Madrid: Estela.

Fernández-Villarán, A. y Rodríguez, A. (2004): Presente y futuro del agroturismo en Vizcaya, Congreso AECIT, Logroño, 2004.

Frey, B. (2000): Arts and Economics, Springer-Verlag.

García, M. (2006): "Veinticinco años de análisis del comportamiento deportivo de la población española (19802005)", en Revista Internacional de Sociología (RIS), Vol. LXIV, núm. 44, mayo-agosto, 15-38.

García, M. (2010): Encuesta sobre hábitos deportivos de los españoles, Madrid: Centro de Investigaciones Sociológicas.

Garcia, M. I. y Fernández \& Zofío, J. L. (2001): "La dimensión económica de la industria de la cultura y el ocio en España: Análisis nacional, regional y sectorial", en Información Comercial Española revista de Economía, núm. 792, págs. 42 a 60.

García, M. I. y Zofío, J. L. (2006): "El impacto económico de la industria de la cultura y el ocio", Adoz: revista de estudios de ocio, ISSN 1134-6019, núm. 29, págs. 35-46.

García, M. I.; Zofio, J. L.; Herrarte, A. y Moral, J. (2009): "La aportación económica de la industria de la cultura y el ocio en España", Estudios de Economía Aplicada, Vol. 27-1, págs. 6186.

González, M. y Benavides, C. (2010): "El gasto en cultura y ocio en España", Documentos 05/2010, EAE business school.

Gratton, C. y Taylor, P. (2000): Economics of sports and recreation, Londres: E\&FN Spon.

Gratton, C. (2004): Sport, health and economic benefith, Sheffield: Sport industry research centre. Sheffield $\mathrm{Ha}$ Ilam University.

Heartfield, J. (2000): Great Expectations: the creative industries in the new economy, Londres: Design.

Heinemann, K. (1998): Introducción a la economía del deporte, Barcelona: Editorial Paidotribo.

INE (2008): El ocio en cifras. Última consulta 23/05/2011.

Instituto de Estudios Turísticos (IET) (2004): Turismo en cifras 2004, Madrid: IET.

Instituto de Estudios Turisticos (IET) (2009): FRONTUR encuesta de movimientos turísticos en fronteras. Informe anual 2009, Madrid: IET.

Instituto de Estudios Turisticos (IET) (2010): Balance del turismo en España en 2010, Madrid: IET.

Instituto de Estudios Turisticos (IET) (2011): FRONTUR encuesta de movimientos turísticos en fronteras. Nota de coyuntura diciembre 2010, Madrid: IET. 
Interactive Software Federation of Europe (2007): Digital entertainment. Who's playing What. Documento web accesible en www.isfe-eu.org. Última consulta 14/05/2011.

Junta de Andalucía (2008): Actividad económica del deporte en Andalucía 2008, Instituto de Análisis Económico y Empresarial de Andalucía.

Kozinets (2010): Netnography, Doing Ethnographic Research Online, Londres, SAGE Publications LTD.

Landabidea, X.; Aguilar, E. y Doistua, J. (2011): "From audience to users and from practice to experience: An approach to television from leisure studies", Second Finnish Transforming Audiences, Transforming Societies Conference: From Audience to Users and Beyond, Aalto University, Espoo.

Lera, F. (2010): "Una aproximación al Deporte desde la Economía. El papel de la Fundación Observatorio Económico del Deporte (FOED)", Encuentro $\mathrm{Na}$ cional de Observatorios del Deporte. 3-5 noviembre, Sevilla.

Lipsey, R. G. (1977): Introducción a la economía positiva, Vicens-Vives.
Mendoza, L. (2011): "Convivencia de políticas de desarrollo económico y sociocultural en los museos de arte contemporáneo", Tesis Doctoral, Universidad de Deusto.

Molitor, G. T. (1999): "The next 1000 years: the big five engines of economic growth", The Futurist, 33 (1), december, págs. 13 a 18.

Nielsen Company (2011): Nielsen Global Consumer Confidence Survey 01 2011. Disponible en http://www. nielsen.com/us/en/insights/reportsdownloads/2011/nielsen-global-consumer-confidence-survey-q1-2011. html?status=success. Última consulta realizada el 26/05/2011.

MCU (2008): Anuario de estadísticas culturales 2008, en www.mcu/es/estadisticas/MC/NAEC/Anuario.htm, última consulta 23/05/2011.

MCU (2010): Anuario de estadísticas culturales 2010, en www.mce.es/estadisticas/MC/NAEC/Anuario.htm, última consulta 23/05/2011.

OCDE (2009): Anuario 2009: Labor Force Statistics, accesible en www.ocde.es/. Última consulta 10/05/2011.
Organización Mundial del Turismo (2008): 2008 International Recommendations for Tourism Statistics, 26-29 February.

Organización Mundial del Turismo (OMT) (1991): "Conferencia Internacional de Estadisticas de Turismo y Viajes", Ottawa, Canadá.

Pedrosa, R. y Salvador, J. A. (2003): "El impacto del deporte en la economía: problemas de medición", Revista Asturiana de Economía, ISSN 1134-8291, núm. 26, págs. 61-84

Pine II, B. J. y Gilmore, J. H. (2002): La Economía de la Experiencia, Gránica, Barcelona.

Sampedro, J. L. (2009): Economía Humanista. Algo más que cifras, Debate, Barcelona.

SpEA (2010): Sport Satillite Accounts, nontechnical methodology paper, commissioned by EC DG Education and Culture, Viena.

Throsby, J. (2001): Economics and culture, Cambridge University Press.

Tribe, J. (1999): Economía del Ocio y del Turismo, Síntesis, Madrid.

UNESCO (2002): Institute for statistics, Montreal. 